\title{
Identification of a 5-microRNA signature and hub miRNA-mRNA interactions associated with pancreatic cancer
}

\author{
XIANXIONG MA ${ }^{1 *}$, RUIKANG TAO $^{2 *}$, LEI LI $^{3 *}$, HENGYU CHEN $^{4}$, ZONGTAO LIU $^{5}$, \\ JIE BAI $^{1}$, XIAOMING SHUAI $^{1}$, CHUANQING WU $^{1}$ and KAIXIONG TAO ${ }^{1}$ \\ ${ }^{1}$ Department of Gastrointestinal Surgery, Union Hospital, Tongji Medical College, \\ Huazhong University of Science and Technology, Wuhan 430022, China; ${ }^{2}$ Department of Biomolecular \\ Engineering, University of California, Santa Cruz, CA 95064, USA; ${ }^{3}$ Department of Breast and Thyroid \\ Surgery, ${ }^{4}$ Department of Pancreatic Surgery, ${ }^{5}$ Department of Cardiovascular Surgery, Union Hospital, \\ Tongji Medical College, Huazhong University of Science and Technology, Wuhan 430022, China
}

Received December 2, 2017; Accepted September 24, 2018

DOI: $10.3892 /$ or.2018.6820

\begin{abstract}
RNA-gene axes have been reported to serve an important role in the carcinogenesis of pancreatic cancer (PC). The aim of the present study was to systematically identity the microRNA signature and hub molecules, as well as hub miRNA-gene axes, and to explore the potential biomarkers and mechanisms associated with the carcinogenesis of PC. Eleven microRNA profile datasets were obtained from the National Center for Biotechnology Information (NCBI) Gene Expression Omnibus (GEO) and ArrayExpress databases, and a meta-analysis was performed to identify the differentially expressed miRNAs (DEMs) between tumor tissue and normal tissue. Subsequently, a diagnostic regression model was constructed to identify PC based on The Cancer Genome Atlas (TCGA) miRNA sequence data by using the least absolute shrinkage and selection operator (LASSO) method. In addition, GSE41368 was downloaded, and a weighted gene co-expression network analysis (WGCNA) was performed to obtain the gene module associated with carcinogenesis by using the TCGAbiolinks and WGCNA packages, respectively. Finally, miRNA-gene networks were constructed and visualized using Cytoscape software, followed by Gene Ontology
\end{abstract}

Correspondence to: Dr Kaixiong Tao or Dr Chuanqing Wu, Department of Gastrointestinal Surgery, Union Hospital, Tongji Medical College, Huazhong University of Science and Technology, Wuhan 430022, China

E-mail: kaixiongtao@hust.edu.cn

E-mail: wucq2014@hust.edu.cn

*Contributed equally

Key words: pancreatic cancer, microRNA, microarray meta-analysis, weighted gene co-expression network analysis, least absolute shrinkage and selection operator, gene set enrichment analysis
(GO) and Kyoto Encyclopedia of Genes and Genomes (KEGG) analyses based on the Database for Annotation, Visualization, and Integrated Discovery (DAVID). A total of 14 DEMs were identified, and a 5-microRNA-based score generated by the LASSO regression model provided a high accuracy for identifying PC [area under the curve $(\mathrm{AUC})=0.918$ ]. In addition, 44 miRNA-mRNA interactions were constructed, and 4 hub genes were screened on the basis of the above bioinformatic tools and databases. Furthermore, 14 biological process (BP) functions and 6 KEGG pathways were identified according to gene set enrichment analysis (GSEA). In summary, the present study applied integrated bioinformatics approaches to generate a holistic view of PC, thereby providing a basis for further clinical application of the 5-miRNA signature and the identified hub molecules, as well as the miRNA-gene axes, which could serve as diagnostic markers and potential treatment targets.

\section{Introduction}

Pancreatic cancer (PC) is the fourth leading cause of cancer mortality, with a 5-year survival rate of $<5 \%$ (1); and even for patients who have undergone radical surgery, the 5-year survival rate is a dismal $15-23 \%$ (2), with a rate of recurrence within 1 year up to $54 \%$ (3). Therefore, an exploration of the underlying mechanisms and identification of novel prognostic markers in order to develop new therapeutic strategies for PC are urgently required.

MicroRNAs (miRNAs), a class of short non-coding RNA molecules that range in size from 19 to 25 nucleotides, have been proposed as promising biomarkers of early cancer detection and as accurate prognosis indicators, as well as targets for more efficient treatment $(4,5)$. miRNAs have important roles in regulating the translation of numerous genes and the degradation of mRNAs through base pairing to partly complementary sites, predominantly in the 3'-untranslated region (UTR) $(6,7)$. Numerous studies have demonstrated that miRNAs serve important roles in the regulation of tumor biology (8-10). Model biomarkers should be easily detectable and correlate 
closely with the clinical outcome, and miRNAs are candidates that may match these criteria.

High-throughput technologies have been employed to identify differences in miRNA and mRNA expression levels between normal and cancerous tissues, and they are increasingly valued as promising tools in medical oncology with a range of clinical applications, i.e., from molecular diagnosis to the molecular classification of cancers, from patient stratification to prognosis prediction, and from novel drug target discovery to tumor response prediction (11-13). Numerous expression profiling studies on PC carcinogenesis have been performed during the last decade using microarray technology, which has revealed hundreds of differentially expressed genes (DEGs) and/or differentially expressed miRNAs (DEMs) to be involved in different pathways and/or biological processes (BPs). Given that independent comparative analyses of the DEGs and DEMs have revealed only a relatively limited reliability for discriminating cancerous from normal tissue (14), it is therefore necessary to conduct meta-analyses to obtain more convincing results. To meet this aim, in the present study DEMs were screened and applied in order to identify a 5-microRNA signature that could be used as a biomarker for PC in The Cancer Genome Atlas (TCGA)-pancreatic adenocarcinoma (PAAD) miRNA sequence datasets using least absolute shrinkage and selection operator (LASSO) (15) regression analysis. Furthermore, weighted gene co-expression network analysis (WGCNA) (16), rather than significance analysis of microarrays (SAM), was used to identify tumor-associated genes. Finally, associations between DEMs and tumor-associated genes were constructed using the Search Tool for the Retrieval of Interacting Genes/Proteins (STRING) (17) database, and visualized using the Cytoscape open source bioinformatics software platform (18).

\section{Materials and methods}

Search strategy and data collection, preprocessing, normalization and integrated analysis. A thorough search of the available literature was performed in the Gene Expression Omnibus (GEO) (https://www.ncbi.nlm.nih.gov/gds) and ArrayExpress electronic databases (https:/www.ebi.ac.uk/arrayexpress/) between January 2007 and October 2017 using the following terms: '[(microRNA OR miRs OR miR OR miRNA) AND (pancreatic OR pancreas)] AND (tumor OR carcinoma OR neoplasm OR cancer)'. Bai and Shuai independently carried out this procedure, and any discrepancies were resolved by mutual discussion.

The inclusion criteria were as follows: i) Original experimental studies that screened for different miRNAs between tumor tissue and normal tissue/adjacent non-tumor tissue in humans; and ii) each dataset contained at least 5 PC samples and 5 normal samples.

The following were the exclusion criteria: i) Duplicated or overlapping studies/datasets; ii) single sample studies and certain platforms with various datasets; iii) laboratory studies/datasets on cell lines, or at the animal level; iv) non-microarray studies/datasets; and v) sequence datasets.

All datasets were extracted using the ArrayExpress and GEOquery packages (19), normalized individually on the base-2 logarithm using the Robust Multi-Array Average and
Linear Models for Microarray (LIMMA) algorithm (20) packages, and annotated by converting different probe IDs into gene IDs. All miRNA names were standardized according to miRBase version 17 via miRBase Tracker (21). Any probes that did not map to a gene ID were removed as viral miRNAs or non-miRNA probes. Subsequently, the MetaDE package (https://cran.r-project.org/src/contrib/Archive/MetaDE/) was applied to integrate the above 11 datasets (data not shown), and filter thresholds were set at $10 \%$, with nPermutation (the number of random permutations to conduct) set at 300 . A P-value and false discovery rate (FDR) of the DEMs $<0.01$ were considered to indicate statistically significant values.

Validation of DEMs by reverse transcription-quantitative polymerase chain reaction ( $R T-q P C R)$. To validate the results of the integrated bioinformatics analysis, 25 pairs of fresh PC and adjacent non-cancerous pancreatic tissues were collected and examined by experienced pathologists at the Union Hospital, Wuhan between April 1 and July 1, 2018. Written informed consent was obtained from all patients or their guardians. The tissue samples were frozen immediately and stored in liquid nitrogen following their surgical resection. Total RNA was extracted using the Qiagen RNeasy ${ }^{\circledR}$ kit (Qiagen $\mathrm{GmbH}$, Hilden, Germany) and subsequently reverse-transcribed into cDNA using an oligo-dT primer and SuperScript II reverse transcriptase (Invitrogen ${ }^{\circledR}$; Thermo Fisher Scientific, Inc., Waltham, MA, USA). Levels of the miRNAs were quantified by RT-qPCR using SYBR ${ }^{\circledR}$ Premix Ex Taq ${ }^{\mathrm{TM}}$ reagent (Takara Bio, Inc., Otsu, Japan) and the ABI7500 real-time PCR system (Applied Biosystems ${ }^{\circledR}$; Thermo Fisher Scientific, Inc.): Denaturation at $94^{\circ} \mathrm{C}$ for $5 \mathrm{~min}$, followed by 36 cycles of denaturation at $94^{\circ} \mathrm{C}$ for $35 \mathrm{sec}$, annealing at $56^{\circ} \mathrm{C}$ for $30 \mathrm{sec}$, and then extension at $72^{\circ} \mathrm{C}$ for $35 \mathrm{sec}$. Relative expression levels were normalized against U6 RNA, and calculated using the $2^{-\Delta \Delta \mathrm{Cq}}$ method (22).

Integrated-signature miRNA analysis of TCGA. In order that the screened DEMs may be used to predict the carcinogenesis of PC, the predicted performance of the DEMs in classifying non-tumor and tumor tissues was estimated based on the TCGA-PAAD miRNA sequence datasets using receiver operating characteristic (ROC) curves. For selection of the significant combined DEMs, the TCGA-PAAD miRNA sequence expression profile data were assessed by applying a LASSO penalized regression analysis method with 10 -fold cross-validation to predict tumor and non-tumor tissue. The best regression model was generated when the minimum $\mathrm{Cp}$ value was chosen. A risk score was generated using the sum of the microRNA expression values weighted by the coefficients from the LASSO regression. Subsequently, the associations between the risk score and the clinical features were estimated. LASSO regression analysis was performed using a 'lars' package based on R software (15). TCGA-PAAD matrix data were extracted using the TCGAbiolinks (23) package.

Weighted gene co-expression network construction and identification of the PC carcinogenesis module. It is necessary to identify the significant coding genes associated with carcinogenesis of PC prior to the construction of miRNA-mRNA networks. The GSE41368 dataset was therefore selected to 


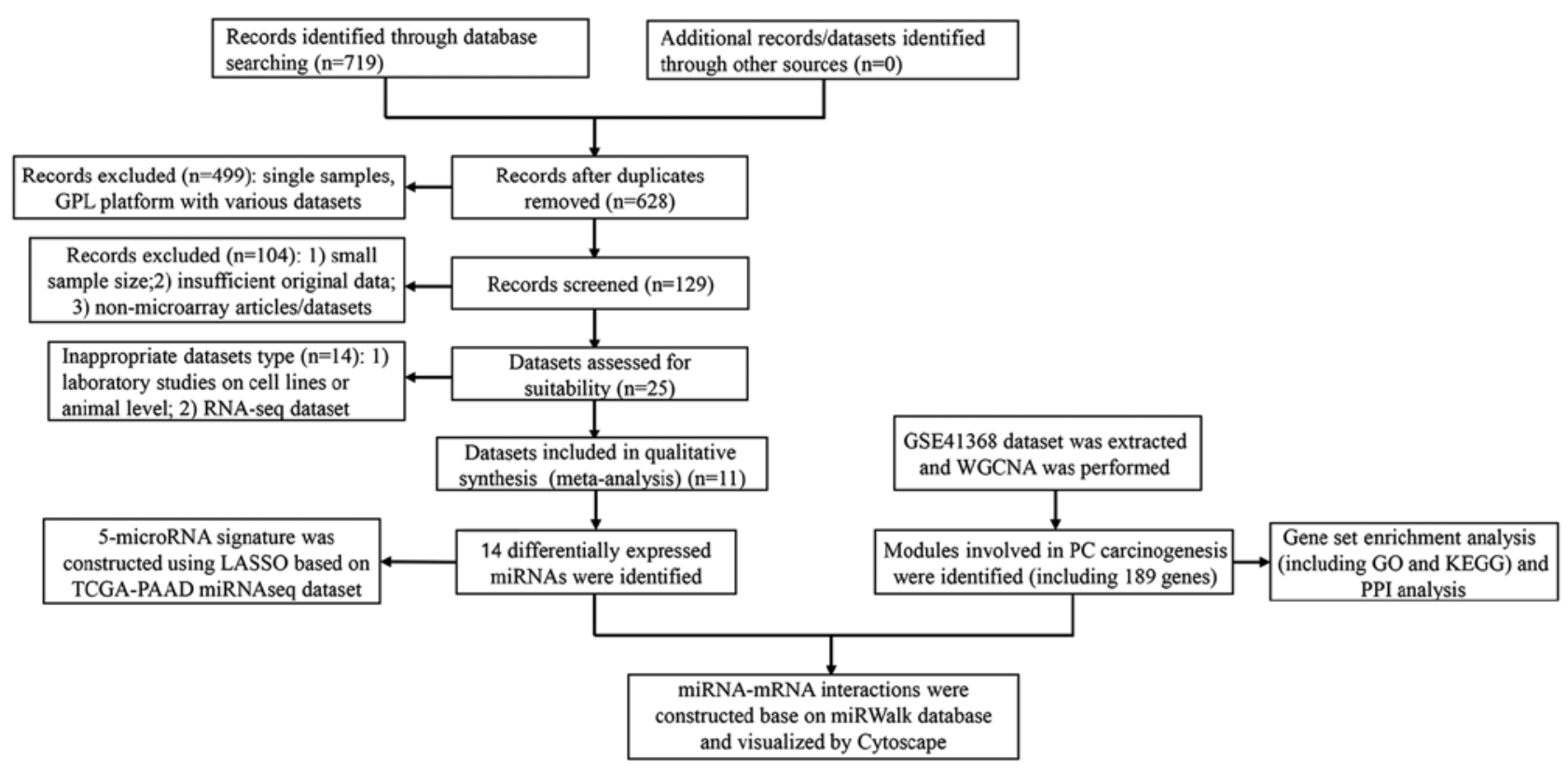

Figure 1. Flow chart of the process for selecting eligible studies in the meta-analysis.

construct the scale-free gene co-expression networks with the WGCNA software package. The expression matrix, containing 20,284 genes and 18 samples, was extracted and normalized using the GEOquery and LIMMA packages, as described above. Subsequently, WGCNA was conducted according to the process proposed by Langfelder et al (16).

The dynamic decision-making tree, node-splitting method and cluster analysis of squared Euclidean distance were used to screen for module eigengenes involved in these clinical traits, particularly those associated with the progression and carcinogenesis of PC. Spearman's correlation analysis was performed to confirm the object module, and the module that had the highest Spearman's correlation coefficient was defined as the carcinogenesis module.

Gene set enrichment analysis (GSEA). To investigate the functions of these gene signatures, Gene Ontology (GO) enrichment analysis based on the GO database was performed, and further assessment of the signaling pathways involved was carried out according to an analysis based on the Kyoto Encyclopedia of Genes and Genomes (KEGG) database. Genes from the carcinogenesis module were applied to the Database for Annotation, Visualization, and Integrated Discovery (DAVID) in order to investigate the biochemical processes and pathways that may be involved in the occurrence and development of the carcinogenesis of PC. Significant categories were identified according to the $\mathrm{P}$-value: The threshold of $\mathrm{P}<0.05$ and a minimum number of genes for the corresponding term $>2$ were considered significant for a category.

miRNA-mRNA network analysis and protein-protein interaction (PPI) network and sub-network analysis. Since DEMs and significant mRNAs involved in the carcinogenesis of PC were identified in the present study, and considering that miRNAs seldom accomplish their functions independently, it was important to identify the interactions of these miRNAs and proteins by researching larger functional groups of miRNAs and proteins (24). The interactions between DEMs and their predicted screened targets were visualized using the Cytoscape open source bioinformatics software platform (18). miRWalk2.0 (25) was utilized to predict the target genes of DEMs, and only the predicted targets obtained simultaneously from miRWalk2.0, TargetScan6.2 (26), miRanda (27) and RNA22 (28) were selected for subsequent PPI analysis. The STRING database was used to annotate functional interactions between genes of the carcinogenesis module, and visualization of the PPI network was also conducted using Cytoscape, version 3.4.0, based on annotation information. A node degree $>4$ was selected as the threshold.

\section{Results}

Integrated analysis of the $11 \mathrm{miRNA}$ expression datasets identified 14 DEMs, 11 of which give rise to statistical significance in the validation test. In total, 64,376 records were identified from the GEO and ArrayExpress databases, respectively. According to the selection criteria, the majority of the preliminarily included entries were eliminated on account of duplicated data, inappropriate article type or inadequate information. Finally, a total of 11 observational studies consisting of 719 cases were retained for subsequent pooling calculation (E-TABM-664, E-MTAB-753, GSE24279, GSE31568, GSE32678, GSE34052, GSE41369, GSE43796, GSE53325, GSE59856, and GSE60978) (data not shown). The study sample sizes ranged from 11 to 250 . All the eligible microarray datasets were shown to satisfy the MIAME (or minimum information about a microarray experiment) principle (29). The selection workflow of all eligible studies in the present meta-analysis is shown in Fig. 1.

In the present meta-analysis, Fisher's method summarizing the $-\log (\mathrm{P}$-value $)$ across studies was applied to merge the datasets, and 300 permutations were conducted to eliminate 
Table I. Integrated analysis of 11 miRNA expression datasets that identified 14 significantly deregulated miRNAs.

\begin{tabular}{lccc}
\hline miRNA & Fisher & P-value & FDR \\
\hline hsa-miR-107 & 204.9924 & $1.00 \mathrm{E}-20$ & $4.40 \mathrm{E}-20$ \\
hsa-miR-375 & 243.8859 & $1.00 \mathrm{E}-20$ & $4.40 \mathrm{E}-20$ \\
hsa-miR-484 & 85.89779 & $1.00 \mathrm{E}-20$ & $4.40 \mathrm{E}-20$ \\
hsa-miR-324-5p & 153.3753 & $1.00 \mathrm{E}-20$ & $4.40 \mathrm{E}-20$ \\
hsa-miR-217 & 154.1077 & $1.00 \mathrm{E}-20$ & $4.40 \mathrm{E}-20$ \\
hsa-miR-429 & 60.76476 & 0.000303 & 0.000952 \\
hsa-miR-184 & 59.9386 & 0.000303 & 0.000952 \\
hsa-miR-422a & 55.78436 & 0.000568 & 0.001376 \\
hsa-miR-498 & 54.68656 & 0.000606 & 0.001481 \\
hsa-miR-409-3p & 54.95649 & 0.000606 & 0.001481 \\
hsa-miR-326 & 53.68486 & 0.000758 & 0.001667 \\
hsa-miR-206 & 50.65474 & 0.002424 & 0.004848 \\
hsa-miR-198 & 45.70564 & 0.008939 & 0.016389 \\
hsa-miR-346 & 40.49565 & 0.02803 & 0.047436 \\
\hline
\end{tabular}

miRNA, microRNA; FDR, false discovery rate.

any significant influence of the large number of samples; furthermore, small expression intensities and small variation (i.e., non-informative) genes were filtered according to the mean and standard deviation (10\%). After having combined the effect size, a total of 14 DEMs were identified (Table I and Fig. 2A and B).

The 14 most deregulated DEMs from the integrated microarray meta-analysis were analyzed by RT-qPCR. Of the 14 DEMs, 11 were revealed to be differentially expressed in PC compared with the para-tumor controls (hsa-miR-107, hsa-miR-375, hsa-miR-484, hsa-miR-324-5p, hsa-miR-217, hsa-miR-429, hsa-miR-498, hsa-miR-409-3p, hsa-miR-326, hsa-miR-346, and hsa-miR-422a) (data not shown).

Construction of the miRNA signature to predict $P C$. The TCGA-PAAD miRNA sequence data were downloaded, and the reads per kilobase per million mapped reads (RPKM) expression matrix was extracted. The expression data of 8 out of 11 of the above DEMs were selected for the following regression analysis, after the removal of miRNAs whose expression level equaled 0 RPKM in any sample. Upon applying the LASSO regression formula (Fig. 2C), the performance of the 5 identified miRNAs in the PC classification was estimated using ROC curve analysis. The combined miRNA panel using the LASSO regression model provided a high classification accuracy of PC [area under the curve (AUC)=0.918] (Fig. 2D); risk score $=8.34 \mathrm{e}^{-6} \mathrm{x}$ hsa.mir. $429+6.69 \mathrm{e}^{-9} \mathrm{x}$ hsa.mir.375-9.97 $\mathrm{e}^{-7}$ x hsa.mir. $217+7.43 \mathrm{e}^{-5} \mathrm{x}$ hsa.mir.107-4.68 $\mathrm{e}^{-4} \mathrm{x}$ hsa.mir.484. hsa. mir.n $=\log _{2}$ (expression of hsa.mir.n).

Clinical features of patients associated with the risk score. The risk score was calculated based on the above formula for each of the observations, and the ones whose risk scores were greater than the median were assigned to the high-risk group, whereas the others were assigned to the low-risk group.
Table II. Relationship of the miRNA risk score to clinical parameters in the pancreatic cancer patients.

\begin{tabular}{|c|c|c|c|c|}
\hline \multirow[b]{2}{*}{ Characteristics } & \multirow[b]{2}{*}{ Total } & \multicolumn{2}{|c|}{$\begin{array}{c}\text { miRNA } \\
\text { score }\end{array}$} & \multirow[b]{2}{*}{ P-value } \\
\hline & & High & Low & \\
\hline Age (years) & 178 & & & 0.098 \\
\hline$\geq 65$ (median) & & 42 & 54 & \\
\hline$<65$ & & 47 & 35 & \\
\hline History of alcohol consumption & 166 & & & 0.23 \\
\hline Yes & & 54 & 48 & \\
\hline No & & 27 & 37 & \\
\hline Anatomic site & 178 & & & 0.717 \\
\hline Head of pancreas & & 71 & 68 & \\
\hline Non-head & & 18 & 21 & \\
\hline Race & 178 & & & 0.82 \\
\hline White & & 77 & 79 & \\
\hline Non-white & & 12 & 10 & \\
\hline Sex & 177 & & & 0.00067 \\
\hline Male & & 60 & 37 & \\
\hline Female & & 28 & 52 & \\
\hline History of diabetes & 146 & & & 1 \\
\hline Yes & & 18 & 19 & \\
\hline No & & 55 & 54 & \\
\hline History of pancreatitis & 142 & & & 1 \\
\hline Yes & & 7 & 7 & \\
\hline No & & 65 & 63 & \\
\hline Vital status & 177 & & & 0.022 \\
\hline Alive & & 51 & 67 & \\
\hline Dead & & 37 & 22 & \\
\hline
\end{tabular}

miRNA, microRNA.

The association between the clinical characteristics of the patients and the risk score is shown in Table II. In the present study, a higher risk score was associated with male gender $(\mathrm{P}=0.00067)$ and dead vital status $(\mathrm{P}=0.022)$, which was in agreement with the 5-miRNA signature diagnosis of PC; however, age, history of alcohol consumption, anatomic site, diabetes history, pancreatitis history and race did not reveal any significance with the risk score.

Co-expression network construction and identification of carcinogenesis modules. A total of 20,284 genes from the GSE41368 dataset were included to construct co-expression networks via WGCNA, after elimination of genes using the method described above in the Materials and methods section. Following selection of the desired samples, the connections between the genes in the gene network were shown to be in line with a scale-free network distribution, where the soft-threshold power $\beta$ was set at 18 (Fig. 3A). The dynamic tree cut method identified modules with similar expression profiles. After the highly similar modules had been merged, a 

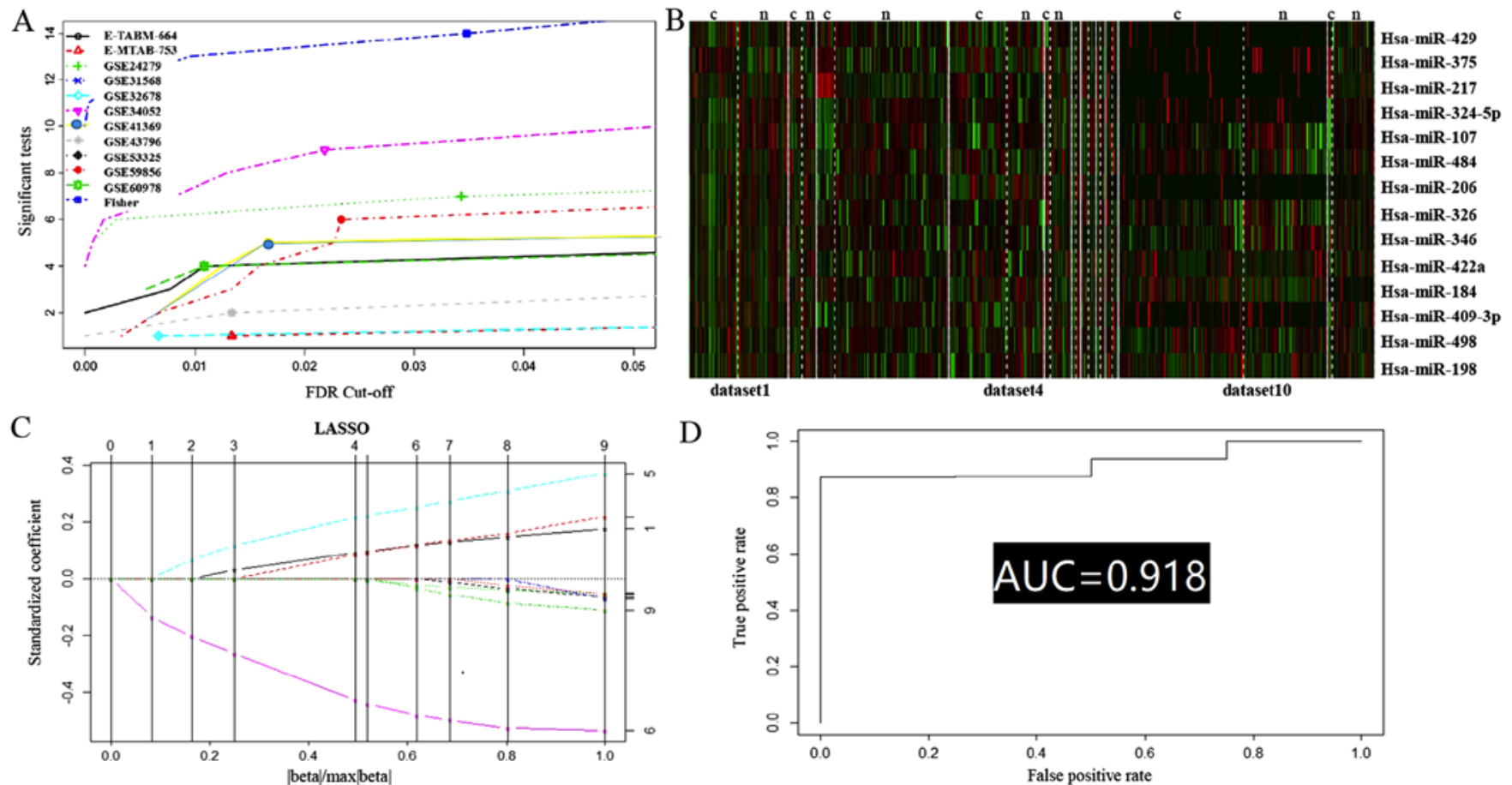

Figure 2. Meta-analysis of the DEMs associated with PC, and the 5-miRNA signature predicts the carcinogenesis of PC. (A) A line chart to plot the number of 14 DEMs against FDR obtained from the meta-analysis by using Fisher's method of combining P-values. (B) A heatmap revealing differential expression profiles of 14 miRNAs detected at a given FDR threshold from the meta-analysis. (C) LASSO coefficient shrinkage analysis was performed to further estimate and select DEMs. Each monotone decreasing curve represents a coefficient of each miRNA. The vertical lines showed whether the coefficients of miRNAs were reduced to zero. (D) The receiver operating characteristic curve for prediction of PC carcinogenesis based on the 5-miRNA risk score level, using corresponding normal tissue as a control (AUC=0.918). DEMs, differentially expressed microRNAs; PC, pancreatic cancer; miRNA, microRNA; FDR, false discovery rate; LASSO, least absolute shrinkage and selection operator; AUC, area under the curve.
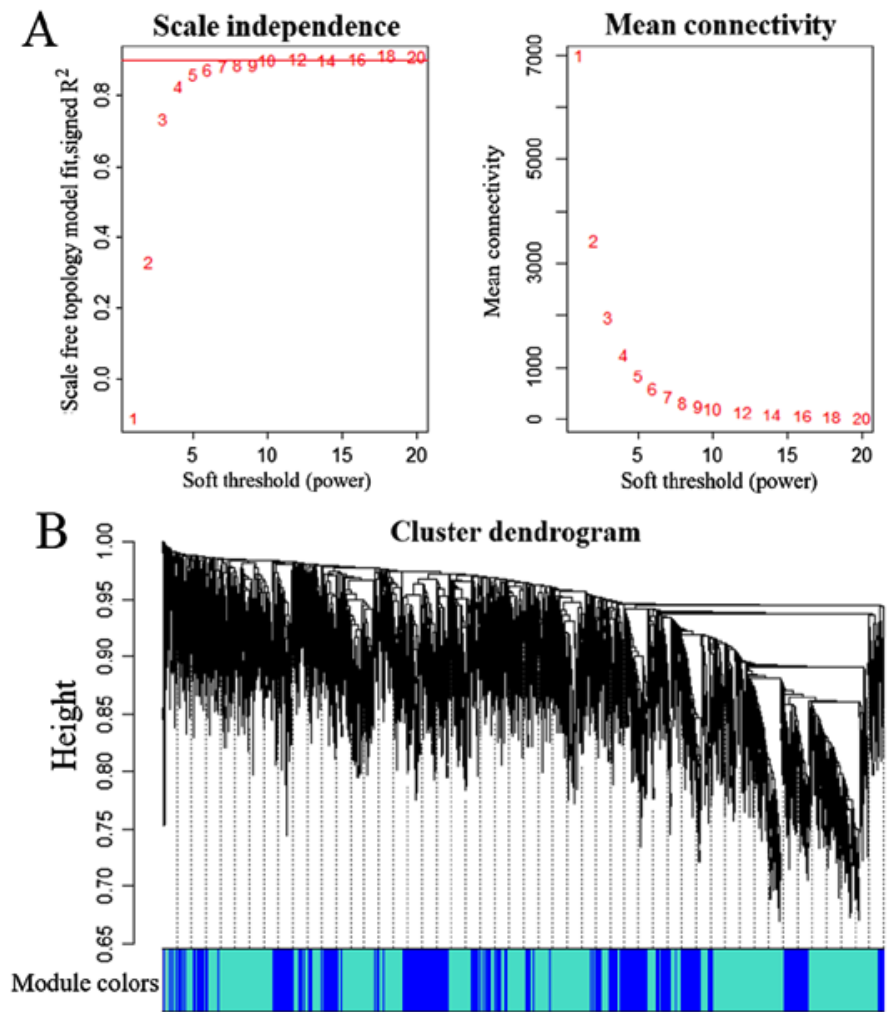

$\mathrm{C}$

Module-trait relationships

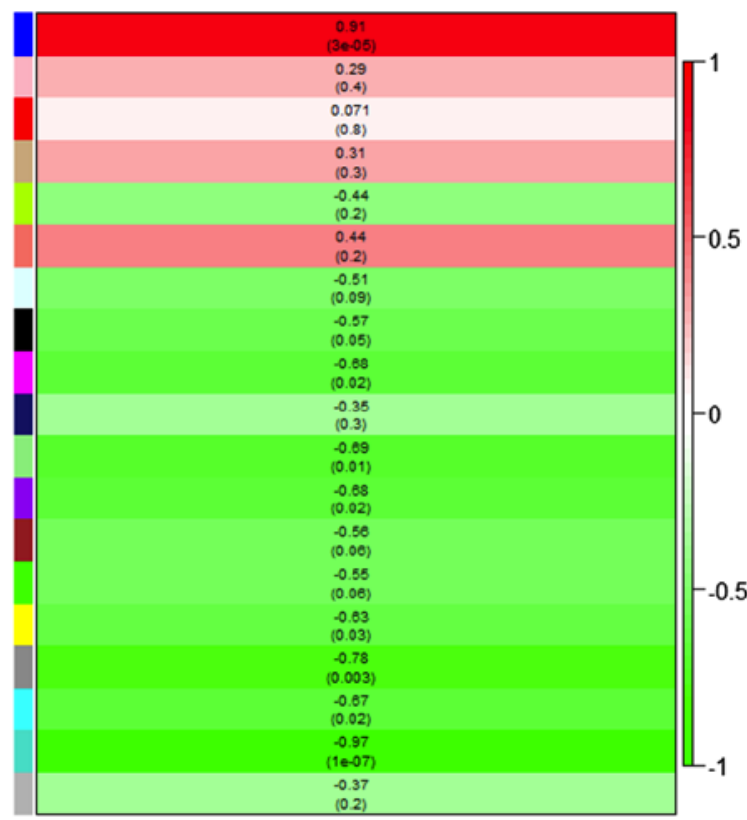

Figure 3. Construction of the weighted gene co-expression network analysis. (A) Scale-free topology criterion of the GSE41368 co-expression network, and an SFT plot for choosing the power $\beta$ for the unsigned weighted correlation network. (B) The hierarchical clustering dendrograms (trees) used for the module identification in the i-th block. (C) Module-trait associations for PC carcinogenesis. The numbers represent Pearson's correlation between clinical traits and the modules. The P-values have been inserted into parentheses, and the color bar shows the correlative strength range from 1 (red) to -1 (green). PC, pancreatic cancer. 
A

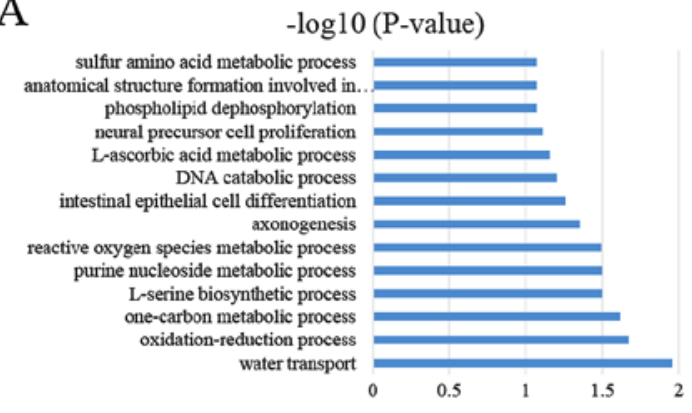

$\mathrm{C}$

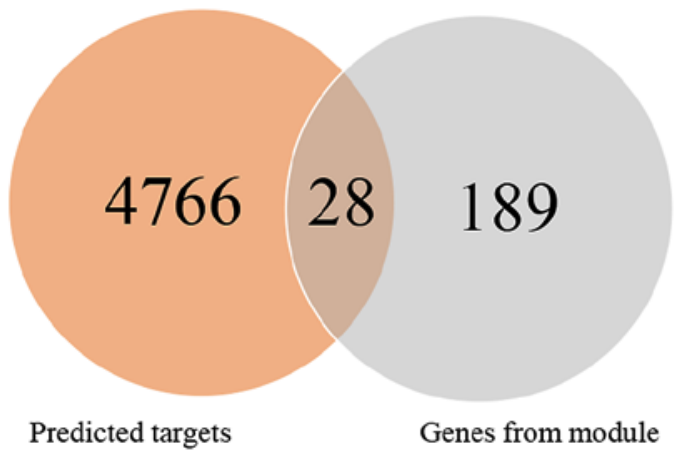

B

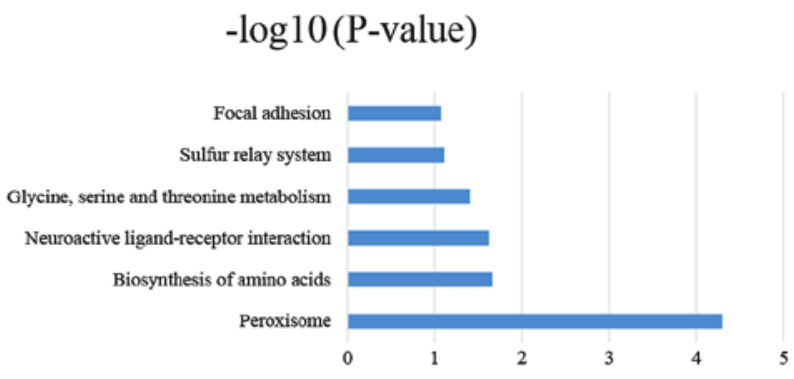

$\mathrm{D}$

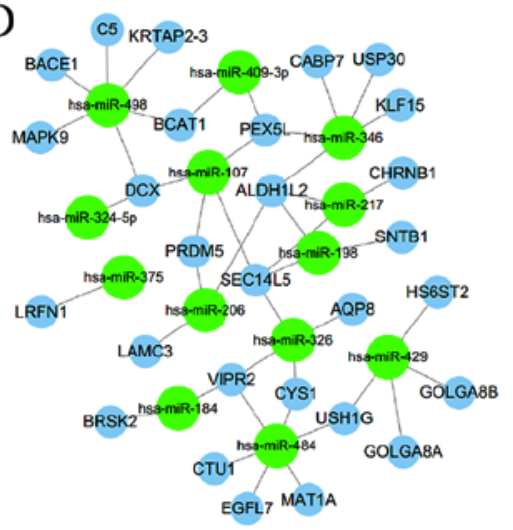

$\mathrm{E}$

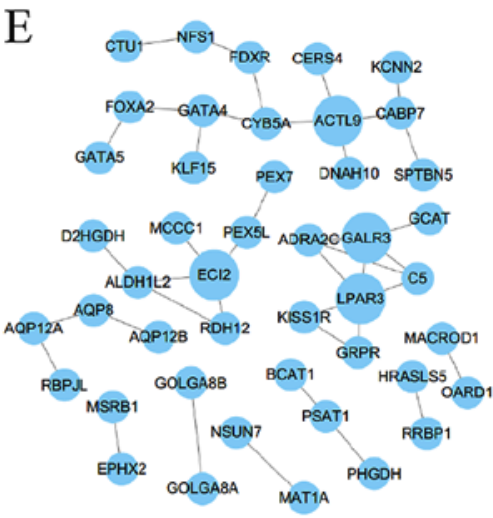

Figure 4. Enrichment analysis of genes from the turquoise module and network visualization. (A) The 14 GO enrichments for 189 module genes are shown. The original significance values were transformed to '- $\log (\mathrm{P}$-value)' in order to plot the curve. (B) The 6 KEGG enrichments for 189 module genes are shown. (C) A Venn diagram illustrating the overlap between the predicted genes of 14 miRNA and module genes. (D) miRNA-mRNA network visualization, where the green and blue nodes represent the miRNAs and their targets, respectively. (E) PPI network visualization. The bigger nodes represent the hub genes. GO, gene ontology; KEGG, Kyoto Encyclopedia of Genes and Genomes; miRNA, microRNA.

total of 18 co-expressed modules were identified, ranging from 148 to 7,255 genes, whereas the 'gray' module was reserved for genes that were not co-expressed (Fig. 3B).

In this type of analysis, it is important to identify modules that have the most significant associations with carcinogenesis of PC. In the present study, the turquoise (189 genes) module yielded the most significant negative Pearson's correlation coefficient (PCC) with the PC carcinogenesis $(r=-0.97$; $\mathrm{P}=1 \mathrm{e}^{-07}$ ) (Fig. 3C).

$G O$ and KEGG analysis. A total of 189 genes from the turquoise module were screened for GSEA. According to the GO analysis, 14 significant enrichments of these top gene signatures were identified, which belonged to the GO BP category. The most significant terms of BP were 'water transport', 'oxidation-reduction process', and 'one-carbon metabolic process'. All the GO terms of BP based on the P-value are shown in Fig. 4A.

Pathway analysis based on the KEGG database revealed that these genes were significantly enriched in 6 terms (Fig. 4B). The most significant terms of KEGG were 'peroxisome', 'biosynthesis of amino acids', and 'neuroactive ligand-receptor interaction'.

miRNA-mRNA and PPI network analysis. A total of 4,766 genes were predicted by using the above 4 algorithms based on the 14 screened DEMs. A total of 28 genes were held in common between those predicted targets and 189 screened genes (Fig. 4C). Subsequently, 14 DEMs and 28 predicted screened targets containing 44 interactions were used to construct miRNA-mRNA networks (Fig. 4D), indicating that those miRNA-mRNA interactions may exert key roles in the carcinogenesis of PC.

The STRING tool was used to obtain the PPI associations of the 189 screened genes, and only interactions with a combined score $>0.4$ were selected to construct networks (Fig. 4E). According to the annotation information from the STRING database, 4 hub genes were identified: ACTL9 (actin-like 9), ECI2 (enoyl-CoA $\Delta$-isomerase 2), GALR3 (galanin receptor 3), and LPAR3 (lysophosphatidic acid receptor 3 ).

\section{Discussion}

PC is a deadly type of cancer, and its occurrence and mortality have been rising worldwide in recent years. The etiology of $\mathrm{PC}$ is unclear due to conflicting evidence, although smoking, obesity and over-consumption of fatty foods are considered to be changeable risk factors $(30,31)$; other unchangeable risk factors include chronic pancreatitis and PC family history $(32,33)$. Understanding the molecular mechanism of PC is of critical importance for diagnosis and treatment. Numerous studies have defined, in part, the microRNA signatures that distinguish patients with PC from normal patients (34), and many microRNAs fulfill their role in PC carcinogenesis by binding to the 3'-UTR of mRNA sequences (35). In the present study, 11 miRNA microarray datasets were integrated to screen for significant DEMs, and the GSE41368 expression matrix was 
subjected to WGCNA in parallel to identify hub modules associated with carcinogenesis. Subsequently, miRNA-mRNA and PPI interactions were constructed to clarify the possible mechanism of PC carcinogenesis, thereby indicating a possible direction for future clinical research.

High-throughput microarray technology has become a popular tool for performing large-scale comparative analyses of gene expression profiles. With the accumulation of bioinformatic data, combining information from numerous similar, already existing studies can improve the reliability and generalizability of results. However, direct combination among heterogeneous datasets is not possible due to the complicated experimental variables embedded in array experiments. Therefore, it is necessary to choose a suitable meta-analysis technique in order to reach convincing conclusions. In the present study, Fisher's inverse Chi-square method based on the MetaDE package was applied to combine the P-values from independent datasets, which is regarded as the most comprehensive approach for meta-analysis of two-class gene expression microarrays (29). As a result, 14 significant DEMs were identified on the condition that $\mathrm{P}<0.01$ and $\mathrm{FDR}<0.01$. Subsequently, clinical samples were collected to perform validation tests, whereupon the majority (11 out of 14) of the DEMs yielded statistically significant results, a finding that was in conformity with our expectation that more reliable results tend to be obtained from integrated meta-analyses.

Increasing evidence has shown that certain miRNAs have critical roles in PC development, and may therefore have potential clinical value in diagnosis, treatment and prognosis evaluation for PC carcinogenesis $(36,37)$. Therefore, TCGA-PAAD miRNA datasets were applied to construct a LASSO regressive model for PC prediction. The LASSO algorithm has numerous advantages compared with ordinary linear regression (38). A linear combination of 5 miRNAs was validated as an independent predictor for $\mathrm{PC}$ carcinogenesis. This signature demonstrated significant diagnostic performance not only in vital status, but also in gender discrimination. The 5-miRNA signature permitted an early diagnosis to be made and precautionary measures in time to be taken, which could prevent further deterioration of the PC. Furthermore, each of the 5 DEMs have been previously reported to serve roles in carcinogenesis (39-42). The results of the present study have helped to corroborate the potential role for miRNAs in the molecular pathogenesis and clinical progression of $\mathrm{PC}$, thereby highlighting the potential of miRNA profiling to improve clinical diagnosis in patients with PC.

Since neither miRNAs nor genes are able to mediate the development of PC independently, it is necessary to identify comprehensive miRNA-mRNA interactions that potentially mediate the pathogenesis of PC. The majority of the studies published previously have solely focused on miRNAs and/or genes to clarify the mechanism of carcinogenesis; only a few of them have identified either DEMs or DEGs independently via a high-throughput method $(43,44)$. Few studies have combined the miRNA expression profiles with those pertaining to mRNAs to explore the potential mechanism. Therefore, the present study was performed to comprehensively identify the most likely miRNA-mRNA interactions, and to elucidate their complex regulatory networks. One notable feature that distinguished the present study from similarly performed studies is that WGCNA was chosen rather than SAM (45) to identify the genes that were most closely associated with PC carcinogenesis, for WGCNA includes not only DEGs, but also those genes that are not significantly differentially expressed, but still have a key role in carcinogenesis.

In the present study, a total of 44 miRNA-mRNA pairs containing 14 dysregulated miRNAs and their 28 target mRNAs were identified. Among the 14 DEMs and 4 hub genes, it was demonstrated that miR-429 is associated with a poor outcome and inhibits pancreatic ductal adenocarcinoma growth by targeting the serine/threonine-protein kinase, TANK-binding kinase 1 (TBK1) (39). Yang et al observed that deregulation of miR-375 inhibited cancer proliferation migration and chemosensitivity in $\mathrm{PC}$ through an association with homeobox B3 (HOXB3) (40). Similarly, chronic pancreatitis and $\mathrm{PC}$ were found to demonstrate an active epithelial-mesenchymal transition profile that is regulated by the miR-217-sirtuin 1 pathway (41), and epigenetic silencing of miR-107 was found to regulate cyclin-dependent kinase 6 expression in PC (42). In addition, active Yes-associated protein (YAP) promoted PC cell motility, invasion and tumorigenesis in a mitotic phosphorylation-dependent manner through LPAR3 (46). Unexpectedly, no gene interactions have been confirmed up to this point. Whether those interactions serve a common role remains unclear, and further experimental validation is required to determine their role in PC carcinogenesis. With the exception of LPAR3, the three other hub genes (ACTL9, ECI2, and GALR3) have been comparatively less well studied in medical science in general, let alone in the oncology field. Exploring gene signatures to predict PC therefore will require further effort.

To the best of our knowledge, differently from other studies, this is the first one performed to date that has utilized the MetaDE package and WGCNA simultaneously to construct miRNA-mRNA interactions to clarify the mechanism of carcinogenesis of PC. The MetaDE package enables the combination of multiple datasets, rather than a single dataset. Similarly, WGCNA takes into consideration all the genes that may exert roles in pathogenesis. More credible results are available with the use of these two tools. However, apart from the promising results thus obtained, limitations with this method do exist, and future applications should be considered. First, only one TCGA-PAAD miRNA sequence dataset and one gene dataset (GSE41368) were enrolled in the present study, which meant that our sample size and sample type were not sufficient to draw entirely reliable conclusions, even though WGCNA was performed. Secondly, other types of molecule, including long non-coding RNAs (lncRNAs) and small interfering RNAs (siRNAs), were not included, which would have enabled a more comprehensive coverage to elucidate the mechanism of PC development. Nevertheless, there were still a number of useful advantages associated with the present comprehensive bioinformatic analysis. The major importance of this study is that it not only provided novel directions for clinical research, but it also provided a way to study the problem itself in clinical practice.

In conclusion, pooled analysis of PC miRNA raw microarrays was performed, and a 5-miRNA signature according 
to the TCGA-PAAD dataset was identified to predict the occurrence of PC against non-tumor tissue. Furthermore, 44 miRNA-mRNA interactions based on 14 DEMs and 28 tumor-associated genes were constructed to illustrate the potential mechanism of $\mathrm{PC}$ carcinogenesis. In addition, $14 \mathrm{GO}$ functions and 6 KEGG pathways were significantly enriched, based on 189 tumor-associated genes. Further large-scale, well-designed and multi-center research studies should be conducted to confirm these findings prior to the application of any predictions, as well as elucidation of the carcinogenesis mechanism of PC.

\section{Acknowledgements}

We sincerely appreciate what our team members have accomplished for these experiments and the accomplishment is the fruit of great effort. The information integrated into this section is correct.

\section{Funding}

The present study was supported by grants from the National Key Basic Research Program of China (no. 2015CB5540007), the National Natural Science Foundation of China (nos. 81472740, 81101825, 81572413, 81600401 and 81702397), and the Hubei Provincial Natural Science Foundation of China (no. 2017CFB474).

\section{Availability of data and materials}

The analyzed datasets generated during the study are available from the corresponding author on reasonable request.

\section{Authors' contributions}

Conceptualization of the study was undertaken by XM, RT and LL. Data collection was performed by JB and XS. Formal analysis of the data was accomplished by XM and HC. Funding acquisition was conducted by $\mathrm{CW}$ and KT. Investigation of the study design was undertaken by XM and RT. Research methodology was designed by XM and LL. Project administration was carried out by KT. Software and analysis was undertaken by XM, RT and ZL. Supervision was undertaken by HC, JB, XS, ZL and CW. Visual images were designed by XM, and $\mathrm{XM}$ wrote the original draft of the manuscript. Further review and editing were carried out by JB, XS, CW and KT. All authors read and approved the manuscript and agree to be accountable for all aspects of the research in ensuring that the accuracy or integrity of any part of the work are appropriately investigated and resolved.

\section{Ethics approval and consent to participate}

This study was approved by the Ethics Committee of Union Hospital, Tongji Medical College, Huazhong University of Science and Technology in compliance with the Helsinki Declaration of 1964 and later versions. Written informed consent was obtained from all the patients prior to their inclusion in the study. This article does not contain any animal studies performed by any of the authors.

\section{Patient consent for publication}

Not applicable.

\section{Competing interests}

The authors declare that there are no competing interests.

\section{References}

1. Jemal A, Siegel R, Ward E, Murray T, Xu J and Thun MJ: Cancer statistics, 2007. CA Cancer J Clin 57: 43-66, 2007.

2. Simianu VV, Zyromski NJ, Nakeeb A and Lillemoe KD: Pancreatic cancer: Progress made. Acta Oncol 49: 407-417, 2010.

3. Hartwig W, Werner J, Jäger D, Debus J and Büchler MW: Improvement of surgical results for pancreatic cancer. Lancet Oncol 14: e476-e485, 2013.

4. Costello E, Greenhalf W and Neoptolemos JP: New biomarkers and targets in pancreatic cancer and their application to treatment. Nat Rev Gastroenterol Hepatol 9: 435-444, 2012.

5. Du Y, Liu M, Gao J and Li Z: Aberrant microRNAs expression patterns in pancreatic cancer and their clinical translation. Cancer Biother Radiopharm 28: 361-369, 2013.

6. Gregory RI, Chendrimada TP, Cooch N and Shiekhattar R: Human RISC couples microRNA biogenesis and posttranscriptional gene silencing. Cell 123: 631-640, 2005.

7. Yates LA, Norbury CJ and Gilbert RJ: The long and short of microRNA. Cell 153: 516-519, 2013.

8. Baer C, Claus R and Plass C: Genome-wide epigenetic regulation of miRNAs in cancer. Cancer Res 73: 473-477, 2013.

9. Singh R and Mo YY: Role of microRNAs in breast cancer. Cancer Biol Ther 14: 201-212, 2013.

10. Song S and Ajani JA: The role of microRNAs in cancers of the upper gastrointestinal tract. Nat Rev Gastroenterol Hepatol 10: 109-118, 2013.

11. Bustin SA and Dorudi S: Gene expression profiling for molecular staging and prognosis prediction in colorectal cancer. Expert Rev Mol Diagn 4: 599-607, 2004.

12. Kulasingam V and Diamandis EP: Strategies for discovering novel cancer biomarkers through utilization of emerging technologies. Nat Clin Pract Oncol 5: 588-599, 2008.

13. Nannini M, Pantaleo MA, Maleddu A, Astolfi A, Formica S and Biasco G: Gene expression profiling in colorectal cancer using microarray technologies: Results and perspectives. Cancer Treat Rev 35: 201-209, 2009.

14. Lascorz J, Hemminki K and Försti A: Systematic enrichment analysis of gene expression profiling studies identifies consensus pathways implicated in colorectal cancer development. J Carcinog 10: 7, 2011.

15. Lu Y, Zhou Y, Qu W, Deng M and Zhang C: A Lasso regression model for the construction of microRNA-target regulatory networks. Bioinformatics 27: 2406-2413, 2011.

16. Langfelder P and Horvath S: WGCNA: An R package for weighted correlation network analysis. BMC Bioinformatics 9: $559,2008$.

17. Franceschini A, Szklarczyk D, Frankild S, Kuhn M, Simonovic M, Roth A, Lin J, Minguez P, Bork P, von Mering C and Jensen LJ: STRING v9.1: Protein-protein interaction networks, with increased coverage and integration. Nucleic Acids Res 41: D808-D815, 2013.

18. Smoot ME, Ono K, Ruscheinski J, Wang PL and Ideker T: Cytoscape 2.8: New features for data integration and network visualization. Bioinformatics 27: 431-432, 2011.

19. Davis S and Meltzer PS: GEOquery: A bridge between the Gene Expression Omnibus (GEO) and BioConductor. Bioinformatics 23: 1846-1847, 2007.

20. Diboun I, Wernisch L, Orengo CA and Koltzenburg M: Microarray analysis after RNA amplification can detect pronounced differences in gene expression using limma. BMC Genomics 7: $252,2006$.

21. Haunsberger SJ, Connolly NM and Prehn JH: miRNAmeConverter: An R/bioconductor package for translating mature miRNA names to different miRBase versions. Bioinformatics 33: 592-593, 2017.

22. Livak KJ and Schmittgen TD: Analysis of relative gene expression data using real-time quantitative PCR and the 2(-Delta Delta C(T)) method. Methods 25: 402-408, 2001. 
23. Colaprico A, Silva TC, Olsen C, Garofano L, Cava C, Garolini D, Sabedot TS, Malta TM, Pagnotta SM, Castiglioni I, et al: TCGAbiolinks: An R/Bioconductor package for integrative analysis of TCGA data. Nucleic Acids Res 44: e71, 2016.

24. Srihari S and Leong HW: Temporal dynamics of protein complexes in PPI networks: A case study using yeast cell cycle dynamics. BMC Bioinformatics 13 (Suppl 17): S16, 2012.

25. Dweep H, Gretz N and Sticht C: miRWalk database for miRNA-target interactions. Methods Mol Biol 1182: 289-305, 2014.

26. Friedman RC, Farh KK, Burge CB and Bartel DP: Most mammalian mRNAs are conserved targets of microRNAs. Genome Res 19: 92-105, 2009.

27. Betel D, Koppal A, Agius P, Sander C and Leslie C: Comprehensive modeling of microRNA targets predicts functional non-conserved and non-canonical sites. Genome Biol 11: R90, 2010.

28. Kertesz M, Iovino N, Unnerstall U, Gaul U and Segal E: The role of site accessibility in microRNA target recognition. Nat Genet 39: 1278-1284, 2007

29. Ramasamy A, Mondry A, Holmes CC and Altman DG: Key issues in conducting a meta-analysis of gene expression microarray datasets. PLoS Med 5: e184, 2008.

30. Yuan C, Morales-Oyarvide V, Babic A, Clish CB, Kraft P, Bao Y, Qian ZR, Rubinson DA, Ng K, Giovannucci EL, et al: Cigarette smoking and pancreatic cancer survival. J Clin Oncol 35: $1822-1828,2017$

31. Carreras-Torres R, Johansson M, Gaborieau V, Haycock PC Wade KH, Relton CL, Martin RM, Davey Smith G and Brennan P: The role of obesity, type 2 diabetes, and metabolic factors in pancreatic cancer: A mendelian randomization study. J Natl Cancer Inst 109: 9, 2017

32. Guerra C, Collado M, Navas C, Schuhmacher AJ, Hernández-Porras I, Cañamero M, Rodriguez-Justo M, Serrano $\mathbf{M}$ and Barbacid M: Pancreatitis-induced inflammation contributes to pancreatic cancer by inhibiting oncogene-induced senescence. Cancer Cell 19: 728-739, 2011.

33. Molina-Montes E, Gomez-Rubio P, Márquez M, Rava M, Löhr M, Michalski CW, Molero X, Farré A, Perea J, Greenhalf W, et al: Risk of pancreatic cancer associated with family history of cancer and other medical conditions by accounting for smoking among relatives. Int J Epidemiol 47: 473-483, 2018.

34. Tanday S: Biomarkers in blood could help to detect pancreatic cancer. Lancet Oncol 15: e108, 2014.

35. Li L, Li Z, Kong X, Xie D, Jia Z, Jiang W, Cui J, Du Y, Wei D, Huang $S$ and Xie K: Down-regulation of microRNA-494 via loss of SMAD4 increases FOXM1 and $\beta$-catenin signaling in pancreatic ductal adenocarcinoma cells. Gastroenterology 147 485-497.e18, 2014

36. Liu R, Chen X, Du Y, Yao W, Shen L, Wang C, Hu Z, Zhuang R, Ning G, Zhang C, et al: Serum microRNA expression profile as a biomarker in the diagnosis and prognosis of pancreatic cancer. Clin Chem 58: 610-618, 2012.
37. Schultz NA, Werner J, Willenbrock H, Roslind A, Giese N, Horn T, Wøjdemann M and Johansen JS: MicroRNA expression profiles associated with pancreatic adenocarcinoma and ampullary adenocarcinoma. Mod Pathol 25: 1609-1622, 2012.

38. Avalos M, Pouyes H, Grandvalet Y, Orriols L and Lagarde E: Sparse conditional logistic regression for analyzing large-scale matched data from epidemiological studies: A simple algorithm. BMC Bioinformatics 16 (Suppl 6): S1, 2015.

39. Song B, Zheng K, Ma H, Liu A, Jing W, Shao C, Li G and Jin G: miR-429 determines poor outcome and inhibits pancreatic ductal adenocarcinoma growth by targeting TBK1. Cell Physiol Biochem 35: 1846-1856, 2015.

40. Yang D, Yan R, Zhang X, Zhu Z, Wang C, Liang C and Zhang X: Deregulation of MicroRNA-375 inhibits cancer proliferation migration and chemosensitivity in pancreatic cancer through the association of HOXB3. Am J Transl Res 8: 1551-1559, 2016.

41. Deng S, Zhu S, Wang B, Li X, Liu Y, Qin Q, Gong Q, Niu Y, Xiang $\mathrm{C}$, Chen $\mathrm{J}$, et al: Chronic pancreatitis and pancreatic cancer demonstrate active epithelial-mesenchymal transition profile, regulated by miR-217-SIRT1 pathway. Cancer Lett 355: 184-191, 2014.

42. Lee KH, Lotterman C, Karikari C, Omura N, Feldmann G, Habbe N, Goggins MG, Mendell JT and Maitra A: Epigenetic silencing of MicroRNA miR-107 regulates cyclin-dependent kinase 6 expression in pancreatic cancer. Pancreatology 9: 293-301, 2009

43. Mao Y, Shen J, Lu Y, Lin K, Wang H, Li Y, Chang P, Walker MG and Li D: RNA sequencing analyses reveal novel differentially expressed genes and pathways in pancreatic cancer. Oncotarget 8: 42537-42547, 2017.

44. Bloomston M, Frankel WL, Petrocca F, Volinia S, Alder H, Hagan JP, Liu CG, Bhatt D, Taccioli C and Croce CM: MicroRNA expression patterns to differentiate pancreatic adenocarcinoma from normal pancreas and chronic pancreatitis. JAMA 297: 1901-1908, 2007

45. Lin D, Shkedy Z, Burzykowski T, Ion R, Göhlmann HW, Bondt AD, Perer T, Geerts T, Van den Wyngaert I and Bijnens L: An investigation on performance of Significance Analysis of Microarray (SAM) for the comparisons of several treatments with one control in the presence of small-variance genes. Biom J 50: 801-823, 2008.

46. Yang S, Zhang L, Purohit V, Shukla SK, Chen X, Yu F, Fu K, Chen Y, Solheim J, Singh PK, et al: Active YAP promotes pancreatic cancer cell motility, invasion and tumorigenesis in a mitotic phosphorylation-dependent manner through LPAR3. Oncotarget 6: 36019-36031, 2015. 